\title{
Micromechanical modeling and experimental characterization of the non linear behavior of platelet-reinforced nanocomposites
}

\author{
Ludovic Cauvin $^{1, a}$, Djimedo Kondo ${ }^{1}$, Mathias Bried ${ }^{1}$ and Naresh Bhatnagar ${ }^{2}$ \\ ${ }^{1}$ Laboratoire de Mécanique de Lille, Boulevard Paul Langevin, 59655 Villeneuve d'Ascq, France \\ 2 Mech. Eng. Dpt., Indian Institute of Technology, HausKhaz, New Delhi, India
}

Received 20 April 2009

\begin{abstract}
Nanocomposites with platelet reinforcements are emergent materials whose deformation mechanisms still need to be carefully investigated. In this study, we first present the studied class of nanocomposites (Polypropylene reinforced by Montmorillonite clay nanoplatelets). From uniaxial tensile tests, it is shown that some mechanical characteristics of the nanocomposite (Young's modulus, yield stress) significantly increase compared to the matrix even for very low volume fraction of reinforcements (maximum of $7 \%$ mass fraction for our tested specimen). The Ponte Castañeda and Willis [6] bound is adopted to account for the evolution of the elastic behavior of the material [7]. Adapting the Hill-type incremental method [13] allows the simulation of the non linear behaviour of the material. However, it is shown that this scheme requires careful choice of the aspect ratio, in the plastic domain, to provide good predictions of plasticity.
\end{abstract}

Key words: Nanocomposites / Polypropylene / Montmorillonite / Experimental study / Non linear homogenization

\begin{abstract}
Résumé - Modélisation micromécanique et caractérisation expérimentale du comportement non linéaire de nanocomposites à renforts plaquettaires. Les nanocomposites à renforts plaquettaires sont des matériaux émergents dont la compréhension des mécanismes de déformation requiert encore des investigations spécifiques. Dans cette étude, nous présentons d'abord brièvement la classe de nanocomposite étudié (Polypropylène renforcé par des nanoplaquettes d'argiles de Montmorillonite). Les essais de traction uniaxiale réalisés sur ce matériau montrent une augmentation significative de différentes caractéristiques mécaniques (module de Young, seuil de plasticité) par rapport à la matrice, y compris pour de faibles taux de renforts ( $7 \%$ de fraction massique au maximum pour nos essais). Le schéma d'homogénéisation de Ponte Castañeda et Willis [6] a été adopté pour rendre compte de l'évolution des propriétés élastiques du matériau [7]. La mise en œuvre d'une adaptation de la méthode incrémentale de Hill [13] permet la simulation du comportement non linéaire du matériau. Cependant, celle-ci nécessite un choix judicieux du rapport d'aspect pour fournir de bons résultats en plasticité.
\end{abstract}

Mots clés : Nanocomposite / Polypropylène / Montmorillonite / Essais mécaniques / Homogénéisation non linéaire

\section{Introduction}

The results obtained by Toyota Research in the 90 's, on the dispersion of nanoscopic platelet silicates (montmorillonite, MMT) in a polyamide 6 matrix have motivated considerable worldwide research in the field

\footnotetext{
a Corresponding author:

ludovic.cauvin@ed.univ-lille1.fr
}

of nanocomposites constituted of thermoset and clay platelets reinforcements $[2,3]$. The remarkable mechanical behavior as well as thermal barrier to gases, etc., for very low mass fraction of reinforcement (less than 5\%), have generated significant industrial and academic interest (see for instance $[1,3,4,15]$ ).

The present study is concerned with an experimental characterization and a micromechanical modeling of the non linear effective behavior of a class of 
platelet-reinforcement nanocomposite. The aim is to better understand the reinforcement effect of nanoscopic platelets. We first describe the studied material which is a polypropylene matrix $(\mathrm{PP})$ reinforced by nanoscopic clay platelets (MMT). After this description, the experimental characterization of the mechanical behavior of the material is performed by means of tensile tests. The elastic behavior is modeled by using two homogenization schemes: Mori-Tanaka [5] estimate and Ponte Castañeda and Willis [6] bound. The implementation of the Ponte Castañeda and Willis [6] bound allows to account separately for the shape of the inclusions and their spatial distribution in the polymeric matrix. The non linear behavior modeling is made by adapting the incremental methods proposed by Hill [13]. Finally, a comparison between experimental data and modeling results is made to estimate the efficiency of the modeling method.

\section{Material description}

The nanocomposite material under investigation is polypropylene (PP) matrix (REPOL H020EG-Reliance make) with Montmorillonite (MMT) clay platelets (CRYSNANO 1010-Southern Clay make). It was manufactured by the Indian Institute of Technology (IIT) of New Delhi in India.

The polypropylene (PP) and the clay (MMT) are first thoroughly mixed for different volume fractions of clay in a co-rotating twin screw extruder (Hake make). The extrudate is converted into granules for molding purposes. The tensile samples are then obtained by injection molding of these nano clay based PP granules. The size of the MMT nano particles is measured with a particle size analyzer (Brookhaven 90Plus) at the IIT. The nano platelets have an ellipsoidal shape with a main diameter of $209 \mathrm{~nm}$, a median diameter of $189 \mathrm{~nm}$ and a thickness of $50 \mathrm{~nm}$ and so can be approximated, if required, spheroidal. Use of X-ray Diffraction (XRD) allows to highlight that the obtained nanocomposite has partially intercalated and partially exfoliated structure. It must be emphasized that according to the processing, three types of material (Fig. 1) can be obtained. In the first type, the nanoclay particles/ platelets are uniformly distributed and dispersed in an aggregate form. This two phase material is expected to enhance mechanical or thermal properties as in classical composites. Therefore, it is classically called micro composite. The second microstructure configuration is composed by intercalated nanocomposites in which polymer chains get into the space between thin platelets of nanometer dimensions, thereby resulting into huge reinforcement. However, in such a case, platelets remain isolated in aggregates between which the matrix is intercalated. The last type is composed by the nanocomposites with exfoliated structure. All the platelets of the aggregates are separated and dispersed within the matrix. The last two microstructure configurations of materials are the true nanocomposites, partially or fully intercalated and/or exfoliated, because the reinforcements are

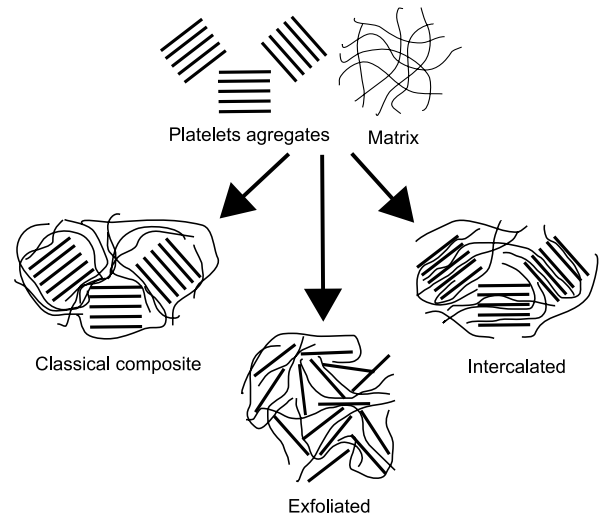

Fig. 1. Different types of nanocomposite.

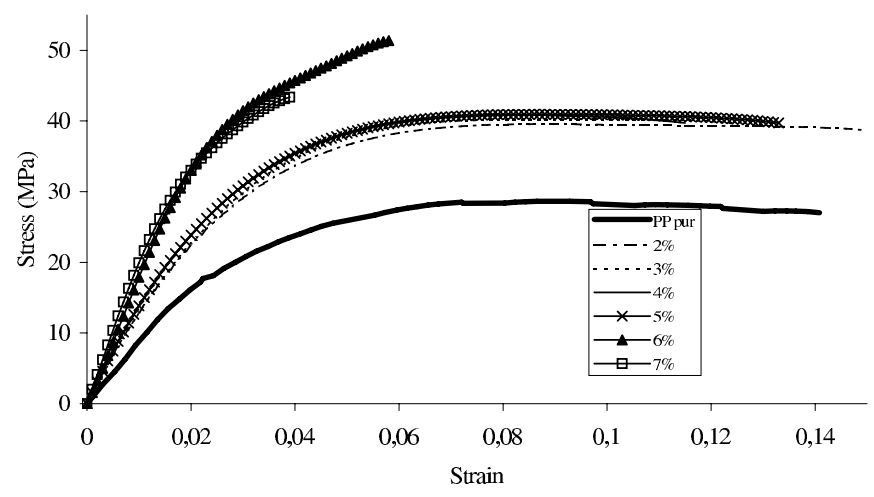

Fig. 2. Tensile test response of the PP-MMT nanocomposite for different MMT weight ratios.

nanoscopics. Note that the most commonly manufactured nanocomposites are those with partially or fully intercalated and/or exfoliated structure [10].

\section{Experimental study of the nanocomposite with platelets reinforcements}

Since experimental data dealing with the mechanical behavior of nanocomposites are not common, uniaxial tensile tests have been conducted on the studied material $(\mathrm{PP}+\mathrm{MMT})$. These tensile tests have been performed on a conventional test machine (INSTRON 4302) with a load cell of $1 \mathrm{kN}$. The strains were measured without contact using a video extensometer (Apollor) that allows the tests to be performed at a constant rate of deformation. For all experiments, this rate of the deformation is taken equal to $10^{-3} \mathrm{~s}^{-1}$ in order to guarantee quasi-static conditions.

All samples shapes and dimensions are based on ASTM D638-03 Type I test and are produced at optimized and constant processing parameters in a numerically controlled injection-molding machine (L\&T Demag make).

Mass fractions of $2 \%, 3 \%, 4 \%, 5 \%, 6 \%$ and $7 \%$ of MMT clay reinforcement embedded in the PP matrix are considered.

Figure 2 shows the response of the material under tensile loading. To ensure the quality of the experimental 


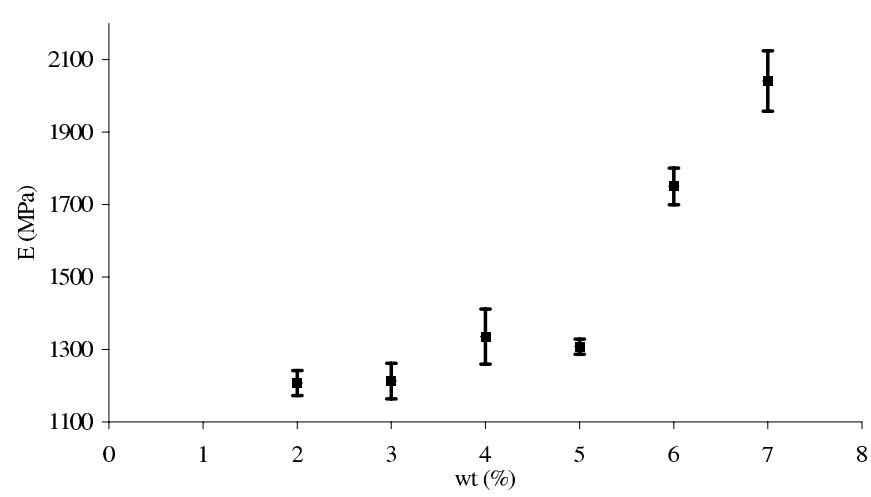

Fig. 3. Young's modulus of the nanocomposite versus the weight ratio $\mathrm{Wt}$ of reinforcements with standard deviations.

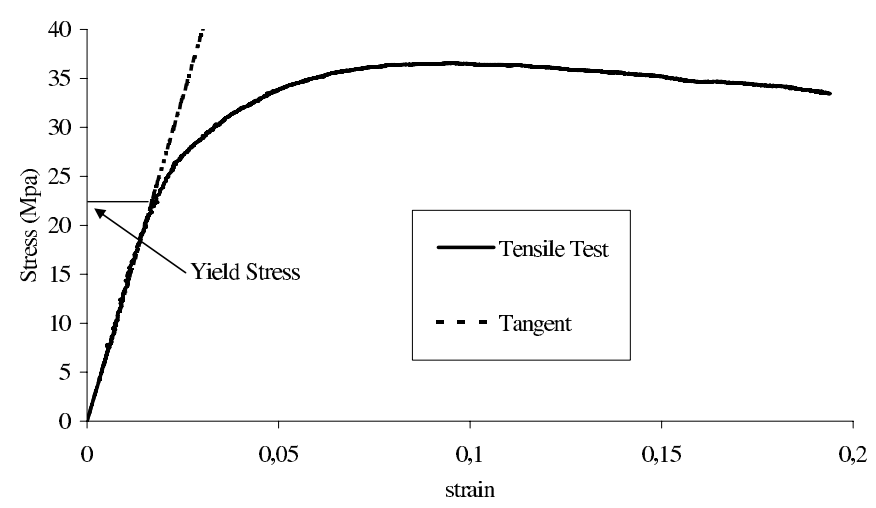

Fig. 4. Determination of the yield stress.

results, several tests have been performed for each reinforcement weight ratio. Results shown in Figure 2 correspond to the average of the mechanical response. The elastic behavior (Young's moduli) has already been the subject of a previous study [7]. A strong effect of the nano platelets on the evolution of the Young modulus with the MMT weight ratio is observed (Fig. 3). For the matrix values, $\mathrm{E}=900 \mathrm{MPa}$ and $\nu=0.4$ are considered. It must be emphasized that the considered weight ratios correspond to very low volume fraction of MMT (less than $3 \%$ ).

From Figure 2, some characteristics of the mechanical behavior of the nanocomposite can be determined: the yield stress, the strain at rupture and the strength. For the determination of the yield stress, we consider that the plastic domain starts at the end of the linear part of the material behavior as shown in Figure 4.

These results, shown in Figure 5, indicate a significant effect of the nano platelet and a quite constant value for the yield stress with MMT reinforcements (about twice greater than that of the matrix). Concerning the strength (Fig. 6), with $2 \%, 3 \%, 4 \%$ and $5 \%$ of MMT clay reinforcement, it is quite constant but increases at $6 \%$ and $7 \%$ of MMT clay reinforcement. For the strain at rupture (Fig. 7), we observe a decrease as the weight ratio increases. These phenomena are probably due to a modification of microstructure, which could be more important between $5 \%$ and $6 \%$ of MMT clay reinforcement.

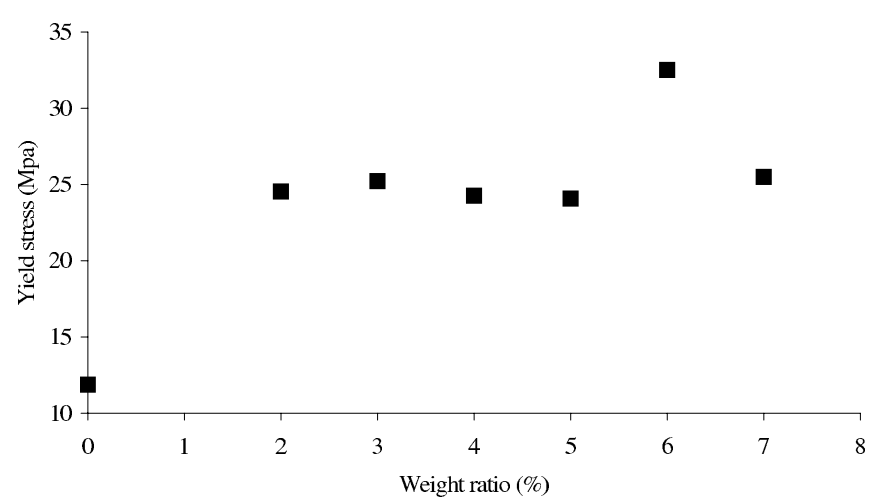

Fig. 5. Yield stress versus platelet weight ratio of reinforcement.

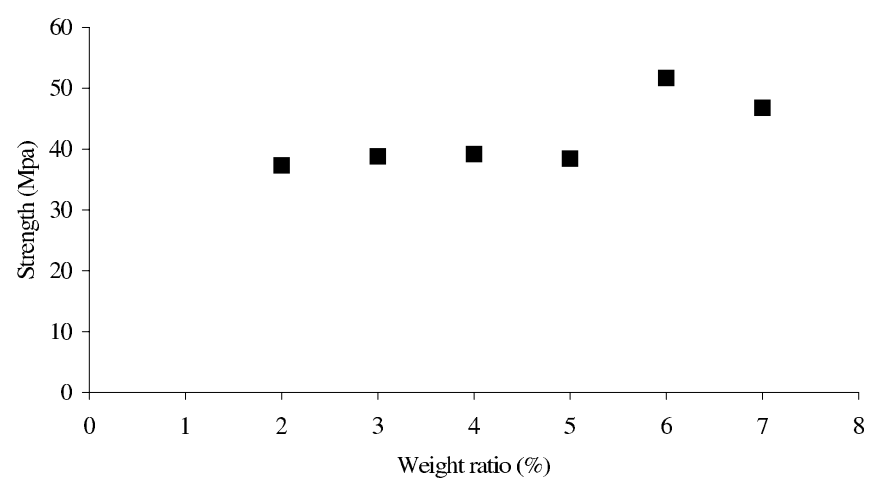

Fig. 6. Strengh versus weight ratio of reinforcement.

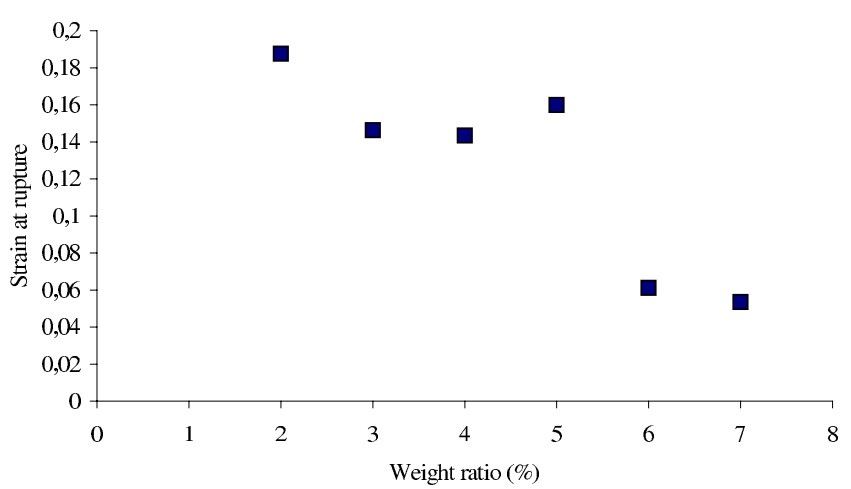

Fig. 7. Strain at rupture versus weight ratio of reinforcement.

\section{Micromechanical modeling and comparison with experimental data}

\subsection{Homogenization of the elastic behavior}

One of the micromechanical models commonly considered for the study of particles-reinforced materials is given by the Mori-Tanaka (MT) estimate [5]. The use of this model by few authors for nanocomposites (see [2]) revealed that it is not completely efficient for this class of materials. According to this observation, our purpose here 
is to consider the Ponte Castañeda and Willis bound [6] which allows to take into account separately the shape of the reinforcements and their spatial distribution in the matrix. This is crucial for the studied material, where reinforcement are platelets with small aspect ratio and the spatial distribution is spherical. Defining the matrix as phase 0 (whose stiffness tensor is denoted $\mathbb{L}^{(0)}$ ) reinforced by $N-1$ families $^{1}$ of inclusions, the Ponte Castañeda and Willis bound provides the following expression of the overall stiffness tensor $\widetilde{\mathbb{L}}$ :

$$
\widetilde{\mathbb{L}}=\mathbb{L}^{(0)}+\left[\mathbb{I}-\sum_{r=1}^{N} f_{(r)} \mathbb{T}^{(r)} \mathbb{P}_{d}\right]^{-1}\left[\sum_{r=1}^{N} f_{(r)} \mathbb{T}^{(r)}\right]
$$

with:

$$
\mathbb{T}^{(r)}=\left[\left(\mathbb{L}^{(r)}-\mathbb{L}^{(0)}\right)^{-1}+\mathbb{P}_{i}^{(r)}\right]^{-1}
$$

$\mathbb{L}^{(i)}$ is the stiffness tensor of the phase $i$ and $f_{(i)}$ the volume fraction of the same phase, the phase $i=0$ being the matrix and $N$ the number of inclusion phases of the considered material ( $N=1$ for a two phase materials).

The fourth-order Hill tensor $\mathbb{P}_{i}$ is associated with the geometry of the inclusions of the considered family, while $\mathbb{P}_{d}$ is associated with the form of the spatial distribution of these inclusions.

Note: Expression (1) allows to retrieve the Mori Tanaka estimate when the $\mathbb{P}$-tensors for the reinforcements geometry and their spatial distribution coincide: $\mathbb{P}_{d}=\mathbb{P}_{i}$.

As the distribution of the orientation of the spheroidal reinforcements is random for the considered materials, the determination of the overall properties requires to perform an average over the orientations. This leads to an isotropic macroscopic stiffness tensor $\widetilde{\mathbb{L}}=3 \widetilde{K} \mathbb{J}+2 \widetilde{\mu} \mathbb{K}$ from which can be determined the compressiblity modulus $\widetilde{K}$ and shear modulus $\widetilde{\mu}$ which in turn allow the evaluation of the Young's modulus $\widetilde{E}$.

Let us also emphasize that the macroscopic strain $\underline{\underline{E}}$ and the local strain $\underline{\underline{\epsilon}}_{r}$ of any phase $r$ is given by:

$$
\underline{\underline{\epsilon}}_{r}=\mathbb{A}^{(r)}: \underline{\underline{E}}
$$

$\mathbb{A}_{(r)}$ is the localization tensor of the strain of the phase $r$. Its expression for the Ponte Castañeda and Willis bound and for the $N-1$ inclusion families reads:

$$
\begin{aligned}
\mathbb{A}^{(r)}= & \left\{\mathbb{I}+\mathbb{P}_{i}^{(r)}:\left(\mathbb{L}^{(r)}-\mathbb{L}^{(0)}\right)\right\}^{-1}: \\
& \left\{f_{(0)} \mathbb{I}+\sum_{j=1}^{N} f_{(j)}\left[\mathbb{I}+\left(\mathbb{P}_{i}^{(j)}-\mathbb{P}_{d}\right)\left(\mathbb{L}^{(j)}-\mathbb{L}^{(0)}\right)\right]\right. \\
& \left.:\left[\mathbb{I}+\mathbb{P}_{i}^{(j)}:\left(\mathbb{L}^{(j)}-\mathbb{L}^{(0)}\right)\right]^{-1}\right\}^{-1}
\end{aligned}
$$

\footnotetext{
${ }^{1}$ Each family of inclusion is characterized by its orientation.
}

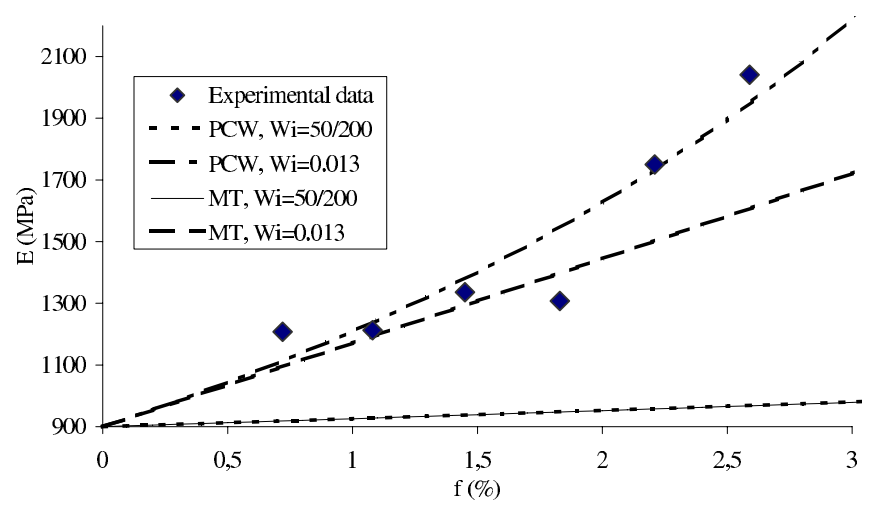

Fig. 8. Comparison between the experimental data and the predictions of the two homogenization estimates (Ponte Castañeda and Willis (PCW) and of Mori Tanaka (MT)) for spheroid reinforcements with the aspect ratio $\frac{50}{200}=0.25$ and 0.013 .

and for the matrix :

$$
\mathbb{A}^{0}=\frac{1}{f_{(0)}}\left(\mathbb{I}-\sum_{r=1}^{N} f_{(r)} \mathbb{A}^{(r)}\right)
$$

\subsection{Comparison between experimental data and elastic modeling results}

In this section, the results obtained, in elasticity, with the Ponte Castañeda and Willis estimate are compared to the experimental data obtained for the studied nanocomposite. The Young's modulus of the matrix is taken as $\mathrm{E}=900 \mathrm{MPa}$ and its Poisson ratio as $\nu=0.4$ (see Sect. 2). According to the dimensions mentioned in Section 2 , the geometry of the platelets is assumed spheroidal with $200 \mathrm{~nm}$ diameter and $50 \mathrm{~nm}$ thickness, thus corresponding to an aspect ratio $W i=\frac{50}{200}=0.25$. As the MMT clay platelets are quite rigid comparatively to the matrix, they are assumed to be elastically rigid. The use of the Ponte Castaeda and Willis bound allows, as already indicated in Section 4.1, to take into account separately the shape of the platelets and their spatial distribution inside the matrix. In agreement with the macroscopic isotropy of the nanocomposite, we assume that the spatial distribution of reinforcements in the matrix is spherical. This assumption seems to be relevant for partially intercalated/partially exfoliated nanocomposites structures. For the spherical spatial distribution of the reinforcements, the isotropic tensor $\mathbb{P}_{d}$ is the one classically associated with a sphere. The modeling results are presented in Figure 8, the Young's modulus evolution is shown as function of the volume fraction of reinforcement. It appears that, by considering $W i=0.25$, both the Mori Tanaka (MT) scheme and the Ponte Castañeda and Willis (PCW) bound predict a modest effect of the reinforcement due to platelets. These results clearly demonstrate that none of the considered model reproduce the experimental data. However, it is worth noticing that measurement of the platelets size is not well controlled because of 
their nanoscopic size. Probably, the measured aspect ratio of 0.25 could be associated to an aggregate in which the matrix is intercalated, and not rigorously to a platelet.

In a first step, it can be expected that a change in the aspect ratio can be used to describe the significant increase of the Young's moduli which also depends on the size of the platelets. From our computations, it was found that a value of $W i=0.013$ allows to obtain a better result for the PCW model while the MT approach still underestimates the overall elastic properties of the considered nanocomposite. In fact, it is known that the results provided by the PCW model for the bulk modulus $\widetilde{K}$ and shear modulus $\widetilde{\mu}$ of the composite have a certain range of validity depending on the concentration and shape of the platelets versus the shape of the spatial distribution of these platelets. Based on the constraints described in [6] (see their formula 4.6) and due to the spherical spatial distribution, it must be mentioned that for these two moduli the results provided by the PCW model are rigorous lower bounds only for platelets volume fractions $f \leq 1,3 \%$ when an aspect ratio of $W i=0.013$ is adopted. Beyond $f=1,3 \%$ these results constitute simply estimates of the macroscopic properties. It can be readily verified that the above observations also applied to the Young's modulus.

\subsection{Principle of the non linear micromechanical modeling}

For the modeling of the non linear behavior of the considered class of materials we adopt the incremental approach proposed by Hill [13] and recently used by different authors for other classes of materials [9, 11, 12].

To this end, the local behavior of each phase $r$ is considered in rate form:

$$
\forall \underline{x} \in(r), \underline{\underline{\dot{\sigma}}}(\underline{x})=\mathbb{L}^{(r)}: \underline{\underline{\dot{\epsilon}}}(\underline{x})
$$

where $\underline{x}$ is the position vector, $\underline{\underline{\dot{\sigma}}}$ the stress rate, $\underline{\underline{\dot{\epsilon}}}$ the strain rate and $\mathbb{L}^{(r)}$ the tangent operator of the phase $r$.

For the implementation of the considered approach, it is assumed that each phase $r$ has an uniform moduli $\mathbb{L}_{(r)}$, although the plastic strain field around and inside the inclusion is expected to be strongly heterogenous. This simplification allows, as classically, to express the macroscopic stress rate as:

$$
\underline{\underline{\Sigma}}=\widetilde{\mathbb{L}}: \underline{\underline{E}}, \text { where: } \widetilde{\mathbb{L}}=\sum_{r=0}^{N} f_{(r)} \mathbb{L}^{(r)}: \mathbb{A}^{(r)}
$$

$\underline{\underline{\underline{\Sigma}}}$ and $\underline{\underline{E}}$ are the macroscopic stress rate and strain rate, $\stackrel{\underline{\underline{\Sigma}}}{\text { respectively. }}$

$\widetilde{\mathbb{L}}$ is the effective tangent operator of the nanocomposite.

\subsection{Comparison between experimental data and non linear modeling results}

For the computation of the Hill incremental method, the matrix behavior is identified from the response shown

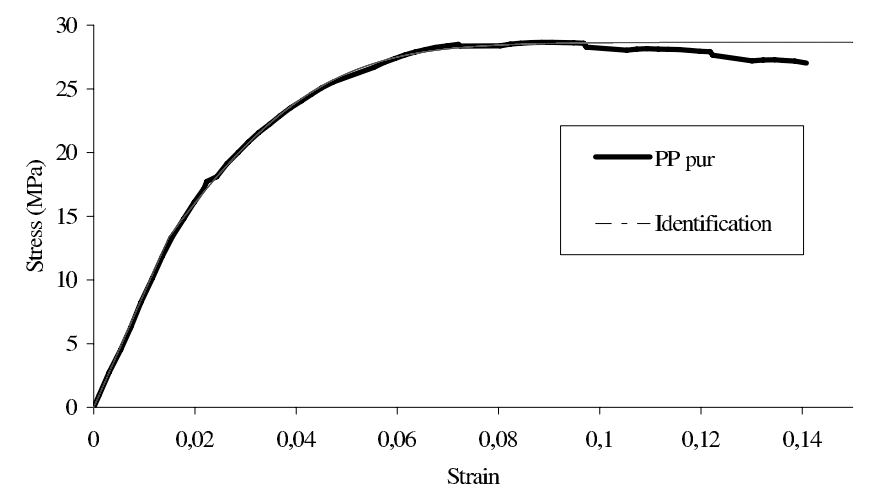

Fig. 9. Polypropylene (PP) matrix identification with the hardening law.

in Figure 2 (Sect. 3) by using a von Mises model. For this purpose, an exponential elastoplastic hardening law is considered:

$$
\sigma^{p}\left(\epsilon^{p}\right)=\sigma_{m}^{p}+\left(\sigma_{0}^{p}-\sigma_{m}^{p}\right) \exp ^{-b \epsilon^{p}}
$$

where $\epsilon^{p}$ is the plastic strain, $\sigma_{0}^{p}$ the yield stress, $\sigma_{m}^{p}$ the asymptotic plastic value of stress and $b$ the rate of plastic hardening.

The yield function reads:

$$
f=\sigma_{e q}-\sigma^{p}
$$

where $\sigma_{e q}$ is the von Mises equivalent stress.

Figure 9 shows the ability of this hardening law to account for the non linear behavior of the PP matrix.

As in [9] (see also [11] and [12]), the implementation of the Hill incremental approach associated to the homogenization scheme with spherical spatial distribution and randomly oriented inclusions, requires an isotropization of the tensor $\mathbb{L}^{(0)}$. The isotropization is given by (see for instance [8]):

$$
\mathbb{L}_{i s o}^{0}=\left(\mathbb{J}:: \mathbb{L}^{0}\right) \mathbb{J}+\frac{1}{5}\left(\mathbb{K}:: \mathbb{L}^{0}\right) \mathbb{K}
$$

where $\mathbb{L}_{i s o}^{0}$ is the isotropic part of $\mathbb{L}^{0}$

$\mathbb{J}_{i j k l}=\frac{1}{3} \delta_{i j} \delta_{k l}, \delta$ being the Kronecker's delta; $\mathbb{K}=\mathbb{I}-\mathbb{J}$

The results provided by the model are shown in Figures $10,11,12,13,14$ and 15 . It is emphasized that, as already observed in the elastic range, the Ponte Castaeda and Willis scheme (PCW) underestimates the reinforcement effect of the platelets when the value 0.25 is taken for the aspect ratio $W i$ (Fig. 8).

Recalling that an aspect ratio $W i=0.013$ appears to be satisfactory in the elastic regime, the modeling of the non linear behavior has been performed with this aspect ratio. It can be observed (Figs. 10, 11, 12, 13, 14 and 15) that the stress is quite overestimated in the plastic domain. These results have led to the search of an aspect ratio able to account for the non linear behavior of the nanocomposite. For the PP reinforced by $2 \%, 3 \%, 4 \%$ and 


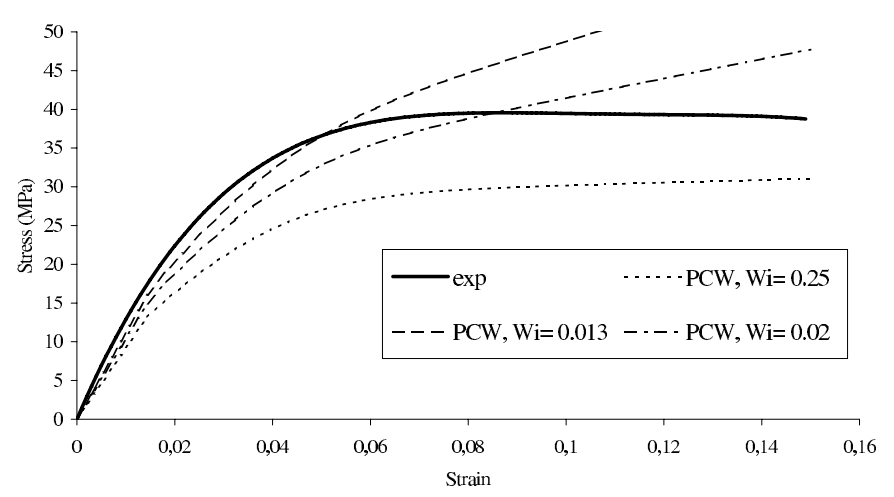

Fig. 10. Comparison between the experimental data and the modeling results for the PP reinforced by $2 \%$ MMT weight ratio for different reinforcement aspect ratios.

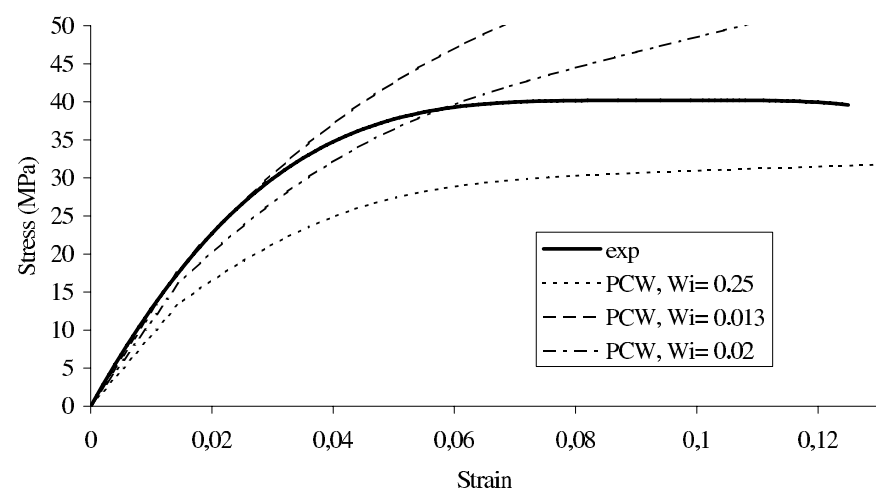

Fig. 11. Comparison between the experimental data and the modeling results for the PP reinforced by $3 \%$ MMT weight ratio for different reinforcement aspect ratios.

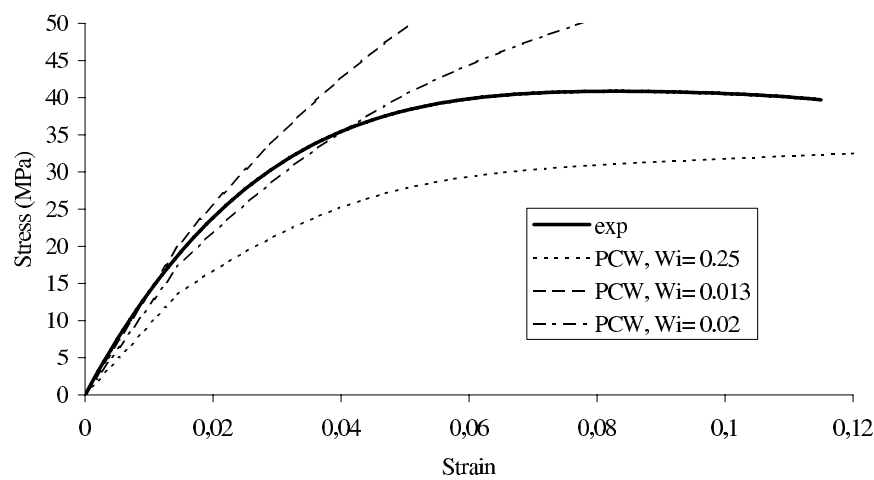

Fig. 12. Comparison between the experimental data and the modeling results for the PP reinforced by $4 \%$ MMT weight ratio for different reinforcement aspect ratios.

$5 \%$ MMT weight ratio, the aspect ratio $W i=0,02$ seems to provide good agreement. For $6 \%$ and $7 \%$ MMT weight ratio, the aspect ratio $W i=0.015$ proved to be convenient. This difference between the two aspect ratios is probably due to particular configuration of microstructure associated to the two ranges of the values.

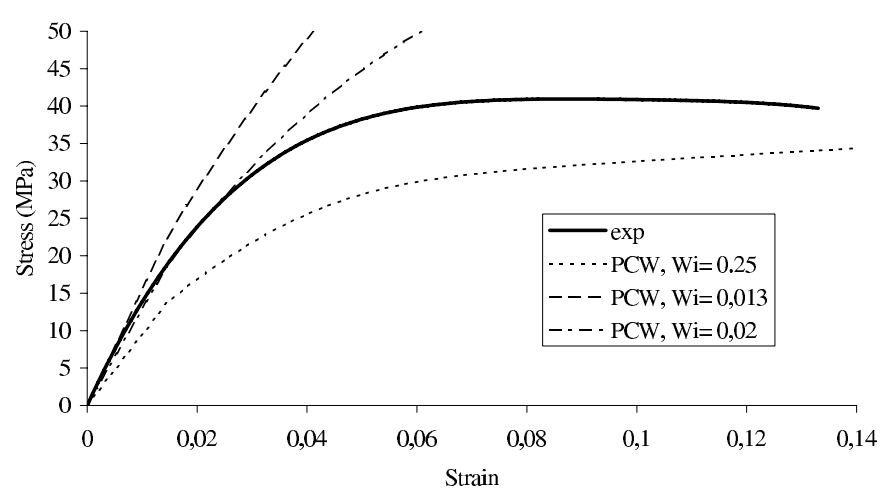

Fig. 13. Comparison between the experimental data and the modeling results for the PP reinforced by $5 \%$ MMT weight ratio for different reinforcement aspect ratios.

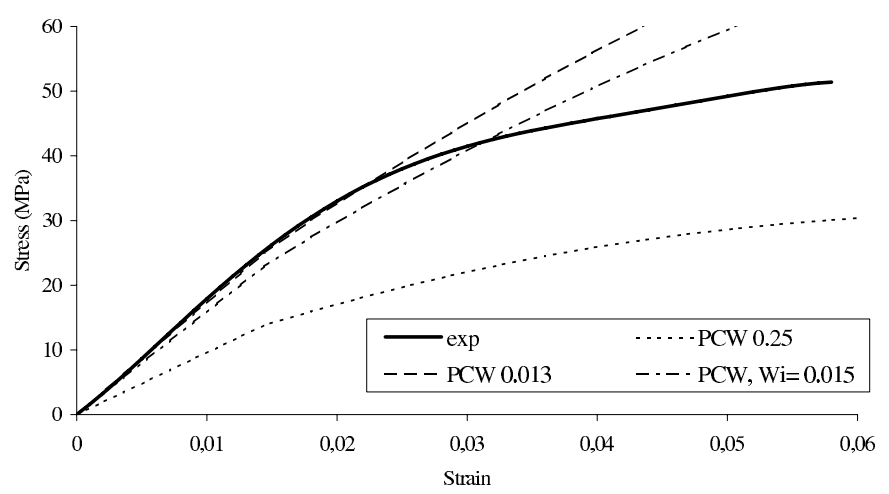

Fig. 14. Comparison between the experimental data and the modeling results for the PP reinforced by $6 \%$ MMT weight ratio for different reinforcement aspect ratios.

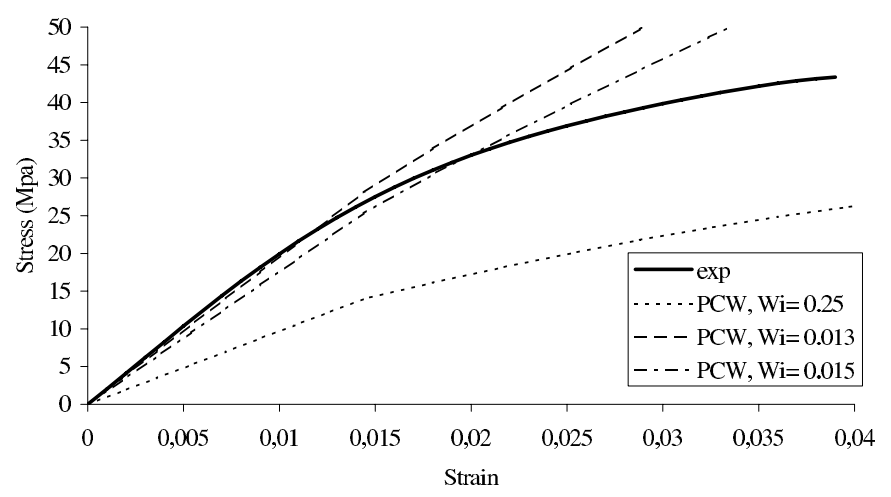

Fig. 15. Comparison between the experimental data and the modeling results for the PP reinforced by $7 \%$ MMT weight ratio for different reinforcement aspect ratios.

\section{Conclusion}

Experimental characterization by tensile tests of a PP reinforced by MMT nano platelets has shown a significant increase of stiffness even for low volume fraction of platelet (less than 3\%). Physical origins of this increase can be found in the shape (platelet with low aspect ratio) and the nanoscopic size of the reinforcements. Modeling of the uniaxial tensile behavior provides good agreement 
with experimental data in elastic regime, but overestimates the stress level in plastic domain. To ensure a good agreement in the whole range of deformation, we have proposed a slight modification of the aspect ratio. Further study of the hardening law may allow to improve the model. Another and more physical way of the model improvement can consist in the study of the modeling of surface effects by introducing an interface (or interphase) $[4,14]$ between matrix and platelet.

\section{References}

[1] E. Chabert, Propriétés mécaniques de nanocomposites à matrice polymère : approche expérimentale et modélisation, Département Génie des Matériaux de l'INSA, Lyon, France, 2002

[2] T.D. Fornes, D.R. Paul, Modelling properties of nylon6/clay nanocomposites using composite theories, Polymer 44 (2003) 4993-5013

[3] J.M. Gloaguen, J.M. Lefebvre, Plastic deformation behaviour of thermoplastic/clay nanocomposites, Polymer 42 (2001) 5841-5847

[4] V. Marcadon, Effets de taille et d'interphase sur le comportement mécanique de nanocomposites particulaires Département Mécanique et Matériaux de l'École Polytechnique, Paris, France, 2005

[5] T. Mori, K. Tanaka, Average stress in matrix and average elastic energy of materials with misfitting inclusions, Acta Metall. 21 (1973) 571-574

[6] P. Ponte Castañeda, J.R. Willis, The effect of spatial distribution on the effective behavior of composite materials and cracked media, J. Mech. Phys. Solids 43 (1995) 1919-1951
[7] L. Cauvin, N. Bhatnagar, M. Brieu, D. Kondo, Experimental study and micromechanical modeling of MMT platelet-reinforced PP nanocomposites, C. R. Mecanique 335 (2007) 702-707

[8] M. Bornert, T. Bretheau, P. Gilormini, Homogénéisation en mécanique des matériaux 1. Matériaux aléatoires élastiques et milieux périodiques, Hermes Sciences Europe Ltd, 2001

[9] I. Doghri, A. Ouaar, Homogenization of two-phase elastoplastic composite materials and structures study of tangent operators, cyclic plasticity and numerical algorithms, Int. J. Solids Struct. 40 (2003) 1681-1712

[10] A. Bafna, G. Beaucage, F. Mirabella, S. Mehtab, 3D Hierarchical orientation in polymer-clay nanocomposite films, Polymer 44 (2003) 1103-1115

[11] J.L. Chaboche, P. Kanouté, On the capabilities of meanfield approaches for the description of plasticity in metal matrix composites, Int. J. Plasticity 21 (2005) 1409-1434

[12] A. Abou-Chakra Guery, F. Cormery, J.F. Shao, D. Kondo, A micromechanical model of elastoplastic and damage behavior of a cohesive geomaterial, Int. J. Solids Struct. 45 (2008) 1406-1429

[13] R. Hill, Continuum Micro-mechanics of elastoplastic polycristals, J. Mech. Phys. Solids 13 (1965) 89-101

[14] P. Sharma, S. Ganti, Size-dependent Eshelby's Tensor for embedded Nano-inclusions Incorporating Surface/Interface Energies, J. Appl. Mech. 71 (2004) 663-671

[15] J.-I. Weon, H.-J. Sue, Effects of clay orientation and aspect ratio on mechanical behavior of nylon- 6 nanocomposite, Polymer 46 (2005) 6325-6334 